\title{
The self-enrichment scenario in intermediate metallicity globular clusters
}

\author{
P. Ventura and F. D’Antona
}

\author{
INAF - Observatory of Rome, via Frascati 33, 00040 MontePorzio Catone (RM), Italy \\ e-mail: [ventura; dantona]@oa-roma.inaf.it
}

Received 25 September 2007 / Accepted 2 November 2007

\begin{abstract}
Context. We present stellar yields computed from detailed models of intermediate mass asymptotic giant branch stars of low metallicity. In this work, all the main microphysics inputs have been updated, and in particular $\alpha$-enhancement is explicitly taken into account in both the opacities and equation of state.

Aims. The target of this work is to provide a basis for testing the reliability of the AGB self-enrichment scenario for globular clusters of intermediate metallicity. These globular clusters exhibit well-defined abundance patterns, which have often been interpreted as the consequence of the pollution of the interstellar medium by the ejecta of massive AGBs.

Methods. We calculated a grid of intermediate-mass models with metallicity $Z=0.001$; the evolutionary sequences are followed from the pre-main sequence throughout the whole AGB phase. We focus our attention on those elements widely studied in the spectroscopic investigations of globular clusters stars, i.e. oxygen, sodium, aluminium, magnesium, and fluorine.

Results. The predictions of our models show an encouraging agreement with the demand of the self-enrichment scenario for what concerns the abundances of oxygen, aluminium, fluorine, and magnesium. The question of sodium is more tricky, due to the large uncertainties of the cross-sections of the Ne-Na cycle. The present results show that only a relatively narrow range of initial masses $\left(M=5,6 M_{\odot}\right)$ can be responsible for the self enrichment.
\end{abstract}

Key words. stars: abundances - stars: AGB and post-AGB - stars: evolution - stars: chemically peculiar - globular clusters: general

\section{Introduction}

Deep spectroscopic investigations in the last decades have shown that globular clusters (GC) stars are not chemically homogeneous samples; instead, they show clear trends involving the chemical abundances of some light elements (Kraft 1994), like magnesium, aluminium, oxygen, fluorine, and sodium. The same behaviour is not followed by halo field stars, which present star-to-star variations limited to carbon and nitrogen, i.e. those elements whose surface abundances are expected to change following the first dredge-up and the mixing possibly following the bump on the red giant branch (hereinafter RGB). In almost the totality of the GCs investigated, a main stellar population is present, whose surface chemistry agrees with the standard $\alpha$-enhanced abundances, and a second component, whose surface abundances of the aforementioned elements define welldetermined patterns (for a recent update, see Carretta 2006; Smith et al. 2005). These stars show depletion of oxygen and fluorine, while the abundances of sodium and aluminium are enhanced with respect to the solar values. The question of a possible depletion of magnesium by $\sim 0.2$ dex is still under debate (Cohen \& Melendez 2005). A common feature of all the GCs investigated is that the $\mathrm{C}+\mathrm{N}+\mathrm{O}$ sum is constant within a factor $\sim 2$ (Ivans et al. 1999; Cohen \& Melendez 2005).

Two explanations, possibly acting together, are currently being investigated to explain such chemical anomalies. a) The first is the deep mixing scenario (DPS), in which a large non canonical extra-mixing, while the stars ascend the RGB, might push the convective envelope inwards, to reach layers where a very advanced nucleosynthesis (full CNO burning) might have acted (Denissenkov \& Weiss 2001; Denissenkov \& Vandenbergh 2003; Denissenkov et al. 1998); b) the second is the selfenrichment scenario (SES), where an early generation of massive AGBs, evolving in the GC, eject into the interstellar medium material that is nuclearly processed, stimulating the formation of a second generation of stars, whose chemistry would trace the composition of the medium out of which they formed (Cottrell \& Da Costa 1981; Ventura et al. 2001) ${ }^{1}$.

The capability of the DPS to fully explain the observed abundance patterns was seriously undermined by the detection of the same chemical anomalies even in main sequence and sub-giant stars of some GCs (Gratton et al. 2001), because the interior of these stars is not expected to reach temperatures high enough to ignite a very advanced nucleosynthesis.

The question concerning the SES is still open, basically because many ingredients used in the stellar evolution theory, whose physical formulation is not directly based on first principles (e.g. convection, mass loss rate, extra-mixing), have a strong impact on the physical and chemical aspects of the AGB evolution (Ventura \& D'Antona 2005a,b). For one of the elements involved in the observed trends, i.e. sodium, the uncertainties associated to the relevant cross-sections ( $\sim 3$ dex, Hale et al. $2002,2004)$ are such to render the results obtained very uncertain (Ventura \& D'Antona 2006). Within the context of the mixing length theory (MLT) modelling of turbulent convection

\footnotetext{
1 Here we do not consider the alternative SES proposed by Maeder \& Meynet (2006), Prantzos \& Charbonnel (2006) and described by Decressin et al. (2007), namely that self-enrichment is produced by the envelopes of fast rotating massive stars.
} 
(Vitense 1953), the theoretical ejecta of AGBs work against the SES hypothesis, because the large number of third dredgeup (TDU) episodes, associated with a modest nucleosynthesis achieved at the bottom of the outer convective envelope, favour a large growth in the overall $\mathrm{C}+\mathrm{N}+\mathrm{O}$ abundances and inhibits the depletion of oxygen (Fenner et al. 2004; Denissenkov \& Herwig 2003). On the other hand, Ventura \& D'Antona (2005b), studying the effects of changing the treatment of convection on the AGB modelling, show that when the full spectrum of turbulence (FST, Canuto \& Mazzitelli 1991) is used to model the convective regions, the FST high efficiency of convection favours higher temperatures at the bottom of the convective envelope, thus an advanced nucleosynthesis (which is usually referred to as "Hot Bottom Burning", HBB), a higher luminosity, and consequently a smaller number of TDU episodes, thus keeping the $\mathrm{C}+\mathrm{N}+\mathrm{O}$ sum almost constant.

An appealing prediction of the SES, and more generally of the role that AGBs may play in the pollution of the interstellar medium within GCs, is that their yields are expected to be helium rich, as a consequence of the deep second dredge up (hereinafter II DUP) experienced particularly by the most massive models. A helium content $Y>0.30$, as found in previous investigations (Ventura et al. 2001), may at least be partially consistent with the existence of helium-rich stellar populations in GCs, which allow the interpretation of the morphology of extended horizontal branches in some GCs (D'Antona et al. 2002; D'Antona \& Caloi 2004; Lee et al. 2005); the presence of a helium-rich population has also been invoked to explain the presence of a blue main sequence in NGC 2808 (D'Antona et al. 2005b; Piotto et al. 2007) and $\omega$ Cen (Bedin et al. 2004; Piotto et al. 2005).

The possibility that DPS and SES might act together to explain the $\mathrm{O}-\mathrm{Na}$ anticorrelation observed in a few giants of M 13 was recently explored by D'Antona \& Ventura (2007), and was previously suggested by Denissenkov et al. (1998) to account for the $\mathrm{Mg}$-Al trend in the same cluster.

Any SES model faces a serious problem: the difficulty of understanding how is it possible that the self-enriched population is very abundant in most of the examined clusters - see, e.g., D'Antona \& Caloi (2007) concerning the ratio of the normal to self-enriched populations derived from the analysis of the horizontal branch (HB) morphology. In particular, for NGC 2808, both the HB - D'Antona \& Caloi (2004), D'Antona et al. (2005) - the main sequence splittings - D'Antona et al. (2005), Piotto et al. (2007) - and the spectroscopic evidence - Carretta et al. (2006) - indicate that about half of the cluster stars are selfenriched. In order to accomplish this we need either: 1) the initial mass function (IMF) of the first generation stars is highly anomalous and peaked at the intermediate-mass stars 2) or the IMF is more or less normal, the initial cluster mass is much higher, by about a factor 10, than the final mass, and the stars of the first generation have been preferentially lost, as discussed, e.g., in D'Antona \& Caloi (2004) and Prantzos \& Charbonnel (2006). In addition, Bekki et al. (2007) suggest that all GCs are formed within dwarf galaxies, so that the cluster formation may take advantage of the infall from all the gas lost by massive AGBs evolving in the galaxy and falling into the protocluster potential well.

In this paper we model the AGB evolution by releasing some of the approximations of our previous works. We focus on an intermediate metallicity $([\mathrm{Fe} / \mathrm{H}] \sim-1.3)$. We postpone the analysis of more metal-rich and metal-poor compositions to forthcoming papers. Our approach is as follows: we present and discuss our intermediate mass models with metallicity $Z=0.001$, which were calculated with the latest and most updated physical inputs. Consistent with the hypothesis we want to test here, we adopt an $\alpha$-enhanced mixture. We compare the yields of the individual elements with the abundances observed in the GCs having appropriate metallicity for the present investigation, taking care to restrict our comparison to those stars that are scarcely evolved, to rule out any possible contamination from canonical or noncanonical extra-mixing that the stars may experience during the RGB evolution. The effects of the uncertainties related to mass loss and the nuclear reaction rates of the $\mathrm{Ne}-\mathrm{Na}$ cycle and the $\mathrm{Mg}-\mathrm{Al}$ chain are also examined.

\section{The ATON stellar evolution code}

The stellar evolution code used in this work is ATON3.1; a full description of the numerical structure can be found in Ventura et al. (1998). Here we briefly recall the main physics inputs, with the most recent updates.

\subsection{Opacities}

We adopt the latest opacities by Alexander \& Ferguson (2005) at temperatures lower than $10000 \mathrm{~K}$ and the OPAL opacities in the version documented by Iglesias \& Rogers (1996). For both the above treatments we have the choice of opacities corresponding either to solar mixture (Grevesse \& Sauval 1998) and to an $\alpha$-enhanced mixture $[\alpha / \mathrm{Fe}]=0.4$. The conductive opacities are taken from Poteckhin (2006, see the web page www.ioffe.rssi.ru/astro/conduct/), and are harmonically added to the radiative opacities.

\subsection{Equation of state}

Tables of the equation of state are generated in the (gas) pressure-temperature plane, according to the method described in D'Antona et al. (2005a). For mixtures including hydrogen, we use the latest OPAL EOS (2005), overwritten in the pressure ionization regime by the EOS by Saumon et al. (1995). The EOS is extended to the high-density, high-temperature domain according to the treatment by Stoltzmann \& Blöcker (2000). After these large tables are written, for a given $Z$, six values of each physical quantity are computed for six different $X$ values. A cubic unidimensional spline provides the interpolation for any input value of $X$. The six tables for $\mathrm{H} / \mathrm{He}$ and given $Z$ are supplemented by 15 tables of $\mathrm{He} / \mathrm{C} / \mathrm{O}$ in which the EOS is directly computed according to Stolzmann \& Blocker (2000), as the non-ionized regions are not present in stellar structure following helium ignition. The interpolation among the 15 tables is performed using triangles in the plane $\mathrm{C} / \mathrm{O}$, as the stechiometric condition is $Y=1-X_{\mathrm{C}}-X_{\mathrm{O}}$.

\subsection{Convection}

The thermodynamic description of those regions that are unstable to convective motions can be addressed either within the context of the traditional MLT formulation (Vitense 1953) or by the FST model (Canuto et al. 1996).

\subsection{Mixing}

Mixing of chemicals within convective zones can be addressed within the instantaneous mixing framework or by a diffusive 
approach. In this case, for each chemical species a diffusive-like equation (Cloutman \& Eoll 1976) is solved:

$$
\frac{\mathrm{d} X_{i}}{\mathrm{~d} t}=\left(\frac{\partial X_{i}}{\partial t}\right)_{\text {nucl }}+\frac{\partial}{\partial m_{r}}\left[\left(4 \pi r^{2} \rho\right)^{2} D \frac{\partial X_{i}}{\partial m_{r}}\right]
$$

where $D$ is the diffusion coefficient, for which, given the convective velocity $v$ and the scale of mixing $l$, a local approximation $\left(D \sim \frac{1}{3} v l\right)$ is adopted.

The borders of the convective regions are fixed according to the Schwarzschild criterium. It is also possible to consider extra mixing by allowing convective velocities to decay exponentially from the formal border, with an e-folding distance described by the free-parameter $\zeta$; see Ventura et al. (1998) for a complete discussion regarding the variation in convective velocities in the proximities of the convective borders.

\subsection{Mass loss}

Mass loss can be treated according to different prescriptions. It is possible to adopt the classic Reimers' treatment, the Vassiliadis \& Wood (1993) formulation, or the prescription given by Blöcker (1995). In the last case, the strong increase in the mass-loss rate during the AGB evolution is modelled by multiplying the Reimers' rate by an ad hoc luminosity power: the final expression is

$\dot{M}=4.83 \times 10^{-22} \eta_{\mathrm{R}} M^{-3.1} L^{3.7} R$

where $\eta_{\mathrm{R}}$ is the free parameter entering the Reimers' prescription.

\subsection{Nuclear network}

The nuclear network includes 30 elements (up to ${ }^{31} \mathrm{P}$ ) and 64 reactions. The full list of the 30 chemicals and of the reactions included can be found in Ventura \& D'Antona (2005a).

The relevant cross sections are taken from the NACRE compilation (Angulo et al. 1999), with only the following exceptions:

1. ${ }^{14} \mathrm{~N}(\mathrm{p}, \gamma){ }^{15} \mathrm{O}$ (Formicola et al. 2004);

2. ${ }^{22} \mathrm{Ne}(\mathrm{p}, \gamma){ }^{23} \mathrm{Na}$ (Hale et al. 2002);

3. ${ }^{23} \mathrm{Na}(\mathrm{p}, \gamma){ }^{24} \mathrm{Mg}$ (Hale et al. 2004);

4. ${ }^{23} \mathrm{Na}(\mathrm{p}, \alpha){ }^{20} \mathrm{Ne}$ (Hale et al. 2004).

\section{The results of the most recent spectroscopic surveys of intermediate metallicity GC}

Several clusters of intermediate metallicity (i.e. $-1.5 \leq[\mathrm{Fe} / \mathrm{H}] \leq$ -1.2) have been investigated spectroscopically in the past decade: among these, the most extensively studied are M3, M 4, M 5, M 13, NGC 6752, and NGC 6218. The first analyses were focused on bright giants, and turn-off (TO) and sub-giant branch (SGB) stars were studied only in the past few years. Bright giants are little help for the main target of this paper, since the observed abundances might be the result of some mixing episode from the bottom of the convective envelope taking place during the RGB evolution; on the other hand, TO and SGB stars never reach internal temperatures high enough to trigger any advanced nuclesynthesis, so any chemical anomaly must have been imprinted in the matter from which they formed. Studies focused on the TO and SGB stars are therefore the most useful for the present investigation.

In the analysis of the observational results, we consider the uncertainties associated to the data and the constant offsets among the different observers, mainly due to differences in the assumed solar abundances and in the temperature scale.

The oxygen-sodium and magnesium-aluminium anticorrelations are by far the most studied trends. For NGC 6752, the works related to TO and SGB stars (Gratton et al. 2001; Carretta et al. 2005) and to bump stars (Gratton et al. 2005), showed a well-defined stellar population in which the oxygen was depleted up to 0.8 dex $([\mathrm{O} / \mathrm{Fe}] \sim-0.4)$ compared to the "standard" abundance $([\mathrm{O} / \mathrm{Fe}] \sim+0.4)$, while sodium was enhanced at most by 0.4 dex. In Gratton et al. (2001) it is also shown that aluminium might be anticorrelated with magnesium, the most peculiar stars showing an aluminium enhancement slightly lower than 1 dex and a magnesium spread of $\sim 0.3$ dex. For this cluster Pasquini et al. (2005) studied the lithium content of TO stars, showing a clear anticorrelation between sodium and lithium, the sodium-rich stars having a lithium content $A(\mathrm{Li})=2^{2}$, a factor of 2 smaller than the value detected in the stars with standard sodium.

The O-Na anticorrelation has recently been confirmed in the investigation of NGC 6218 performed by the same group (Carretta et al. 2006): for the stars below the RGB bump, the authors find a maximum extent of the oxygen depletion by $\sim 1 \mathrm{dex}$, and a maximum sodium enhancement of 0.5 dex.

Ramirez \& Cohen (2003) spectroscopically analysed stars belonging to M 5. For the stars near the TO, they found a stellar population where sodium was enhanced up to $[\mathrm{Na} / \mathrm{Fe}]=0.3 \mathrm{dex}$, while for oxygen they could only find upper limits for the estimated abundances. This is the main reason the authors could not detect any clear $\mathrm{Na}-\mathrm{O}$ anticorrelation.

The case of M4 is hard to analyse, as only red giants spectra are available. A first extensive exploration by Ivans et al. (1999) showed an anticorrelation among the $\mathrm{CN}$ weak stars, with a depletion of oxygen by $\delta[\mathrm{O} / \mathrm{Fe}] \sim 0.6 \mathrm{dex}$, and an enhancement in sodium up to $[\mathrm{Na} / \mathrm{Fe}]=0.3 \mathrm{dex}$. The most oxygen-poor stars in this subsample are aluminium rich $([\mathrm{Al} / \mathrm{Fe}]=0.8 \mathrm{dex})$. The magnesium abundance is constant within 0.2 dex. A more recent work by Smith et al. (2005), unfortunately restricted to giants, confirmed the extent of the oxygen depletion (taking a clear constant offset into account, which may be understood in the leftupper panel of their Fig. 4) and of the aluminium enhancement found by Ivans et al. (1999). They also found a clear anticorrelation between sodium and fluorine, the most sodium-rich stars being depleted in fluorine by $[\mathrm{F} / \mathrm{Fe}]=-0.8$ dex.

Both M 3 and M 13 have been extensively studied in the literature. Sneden et al. (2004) show a clear O-Na anticorrelation, which, for the high gravity stars (i.e. those well below the RGB bump), extends to $[\mathrm{O} / \mathrm{Fe}] \sim-0.4$, and $[\mathrm{Na} / \mathrm{Fe}]=+0.4$. They also confirm, even for this high gravity population, that the most oxygen-poor and sodium-rich stars are largely aluminium enhanced, with $[\mathrm{Al} / \mathrm{Fe}]=1$. The authors also claim detection in both clusters of a magnesium - aluminium anticorrelation, but this result has been recently argued by Cohen \& Melendez (2005), who confirm the extent of the oxygen depletion found by Sneden et al. (2004), but also limit the sodium enhancement for the stars below the RGB bump to $[\mathrm{Na} / \mathrm{Fe}]=+0.3$.

On the basis of the results quoted above, we may summarise the following chemical features which characterise the stars in the GCs that should have not experienced any "in situ" mechanism to change their surface abundances:

1. the oxygen depletion is at most $\delta[\mathrm{O} / \mathrm{Fe}] \sim 0.8$;

2. the most aluminium-rich stars show up an ${ }^{27} \mathrm{Al}$ abundance of $[\mathrm{Al} / \mathrm{Fe}]=1$;

${ }^{2}$ We use the notation $A(\mathrm{Li})=\log (\mathrm{Li} / \mathrm{H})+12$ (in number). 
Table 1. Evolutionary properties of intermediate-mass models.

\begin{tabular}{cccccccccc}
\hline \hline$M / M_{\odot}$ & $\tau_{\mathrm{H}} / 10^{6}$ & $\tau_{\mathrm{He}} / 10^{6}$ & $\tau_{\mathrm{AGB}} / 10^{3}$ & $\delta\left(M_{2 \text { dup }}\right)$ & $M_{\text {core }} / M_{\odot}$ & $\log \left(L / L_{\odot}\right)_{\max }$ & $T_{\text {max }}^{\text {bce }}$ & $N_{\text {pulse }}(\mathrm{TDU})$ & $\lambda$ \\
\hline 3.0 & 275 & 49 & 410 & 0.01 & 0.76 & 4.40 & 74 & 6 & 0.7 \\
3.5 & 195 & 31 & 340 & 0.05 & 0.80 & 4.50 & 83 & 6 & 0.7 \\
4.0 & 145 & 21 & 250 & 0.15 & 0.83 & 4.60 & 88 & 8 & 0.7 \\
4.5 & 112 & 16 & 190 & 0.27 & 0.86 & 4.67 & 95 & 9 & 0.6 \\
5.0 & 91 & 12 & 120 & 0.36 & 0.90 & 4.74 & 100 & 13 & 0.5 \\
5.5 & 75 & 9.5 & 85 & 0.47 & 0.94 & 4.80 & 110 & 17 & 0.5 \\
6.0 & 63 & 7.7 & 42 & 0.56 & 1.00 & 4.90 & 110 & 20 & 0.3 \\
6.3 & 57 & 7.0 & 32 & 0.62 & 1.03 & 4.95 & 112 & 21 & 0.2 \\
\hline
\end{tabular}

3. sodium is anticorrelated to oxygen. The extent of the largest sodium enhancement is $[\mathrm{Na} / \mathrm{Fe}]=0.4$, but this result is uncertain by at least 0.1 dex.

Also, we may mention other two points, which must still be confirmed by detailed spectroscopic investigations:

1. magnesium might be correlated to oxygen, and anticorrelated to sodium and aluminium;

2. fluorine might be strongly anticorrelated to sodium, but this result has been found at the moment only for giant stars;

3. lithium is correlated to oxygen: the oxygen-poor population shows a smaller lithium content by a factor 2 .

\section{The physical properties of the intermediate-mass models}

The models were evolved from the pre-MS through the whole AGB phase. The adopted chemistry is typical of the intermediate metallicity GCs, i.e. $Z=0.001$ and $Y=0.24$. The mixture, consistent with the main target of this investigation, is assumed to be $\alpha$-enhanced, with $[\alpha / \mathrm{Fe}]=+0.4$; the individual abundances are taken from Grevesse \& Sauval (1998). The range of masses involved is $3 M_{\odot} \leq M \leq 6.3 M_{\odot}$, and the limits were chosen to restrict the analysis to stars achieving HBB during the AGB phase and not undergoing any carbon ignition in the interior. Convection was modelled according to the FST treatment. Due to the importance that CNO-burning within the most internal part of the convective envelope may have on the AGB evolution (Herwig 2005; Mazzitelli et al. 1999), we adopted the diffusive treatment in all the models presented here. In the phases preceeding the AGB evolution, a free parameter $\zeta=0.02$ was used to model the exponential decay of velocities within regions that are stable against convection. This agrees with the calibration in Ventura et al. (1998). For the whole AGB phase, no extramixing was assumed from any convective border. Mass loss was modelled according to Blöcker (1995), with the free parameter entering Eq. (2) set to $\eta_{\mathrm{R}}=0.02$, according to the calibration given in Ventura et al. (2000). The main physical properties of the models are reported in Table 1 . Columns 2 to 4 show the duration of the core $\mathrm{H}$ - and He-burning phases (Myr) and of the AGB phase (Kyr).

Within stars of intermediate mass, after the extinction of the CNO-burning shell following the core He-burning phase, the bottom of the convective envelope sinks inwards, down to layers previously touched by nuclear burning. This is the second dredge-up (Iben 1991) Col. 5 reports the total mass previously involved in CNO-burning mixed with the convective envelope during the II DUP. In the most massive models, where the amount of dredged-up matter is higher, the surface chemistry is altered, with a decrease in the surface oxygen abundance and an increase in the sodium and helium mass fractions.
These results are consistent with the old models calculated with the same metallicity as presented in Ventura \& D'Antona (2005b, see Table 1). In Cols. 6-10 of Table 1, we give some details of the AGB evolution, namely the core mass at the beginning of the thermal pulses (TPs) phase, the maximum luminosity and the maximum temperature reached at the bottom of the convective zone $\left(T_{\text {bce }}\right)$, the number of the first TP followed by a TDU episode, and the maximum efficiency of the TDU during the whole stellar AGB life ${ }^{3}$.

Figure 1 shows the variation with mass (decreasing during the evolution) of the luminosity and $T_{\text {bce }}$ in some of our models. We note in all cases, with the only possible exception of the $3 M_{\odot}$ model, a rapid increase in the luminosity after the very first TPs, associated to an increase in $T_{\text {bce }}$. This is a consequence of the high efficiency of the convective model adopted, as correctly predicted by Blöcker \& Schönberner (1991) and later confirmed by D'Antona \& Mazzitelli (1996) and Ventura et al. (2000). A detailed comparison of the results obtained with various efficiencies of the convective model can be found in Ventura \& D'Antona (2005b).

The high luminosities attained by our models have two important consequences:

1. a fast decrease in the mass of the envelope, with a consequent small number of TPs, and therefore of TDUs;

2. a very advanced nucleosynthesis at the bottom of the envelope.

In the most massive models, mass loss is so great that they reach their maximum luminosity after a few TPs. They lose their envelope so rapidly that TDU takes place only in the latest stages of their AGB evolution and with very modest efficiency (see Col. 10 of Table 1). We stress that the higher is the luminosity, the faster the general cooling of the structure determined by the gradual loss of the mass of the envelope. The maximum temperature achieved at the bottom of the external convective zone reaches a maximum asymptotic value (see Col. 8 of Table 1), which, for large $M$, turns out to be independent of the total initial mass of the star; in the present computations, this upper limit is $T_{\text {bce }}=110 \mathrm{MK}$.

\section{The chemistry of the ejecta}

For each model, we calculated the average mass fractions in the ejecta for the chemical elements included in our network. The results for the species of interest for this work are presented in Table 2. For any isotope A, we give the quantity $[\mathrm{A} / \mathrm{Fe}]$, defined

\footnotetext{
3 We use here the usual descriprion of the efficiency of the TDU in the terms of $\lambda$, defined as the ratio of the total matter dredge-up after a TP to the mass by which the H-exhausted core advanced from the previous TP.
} 

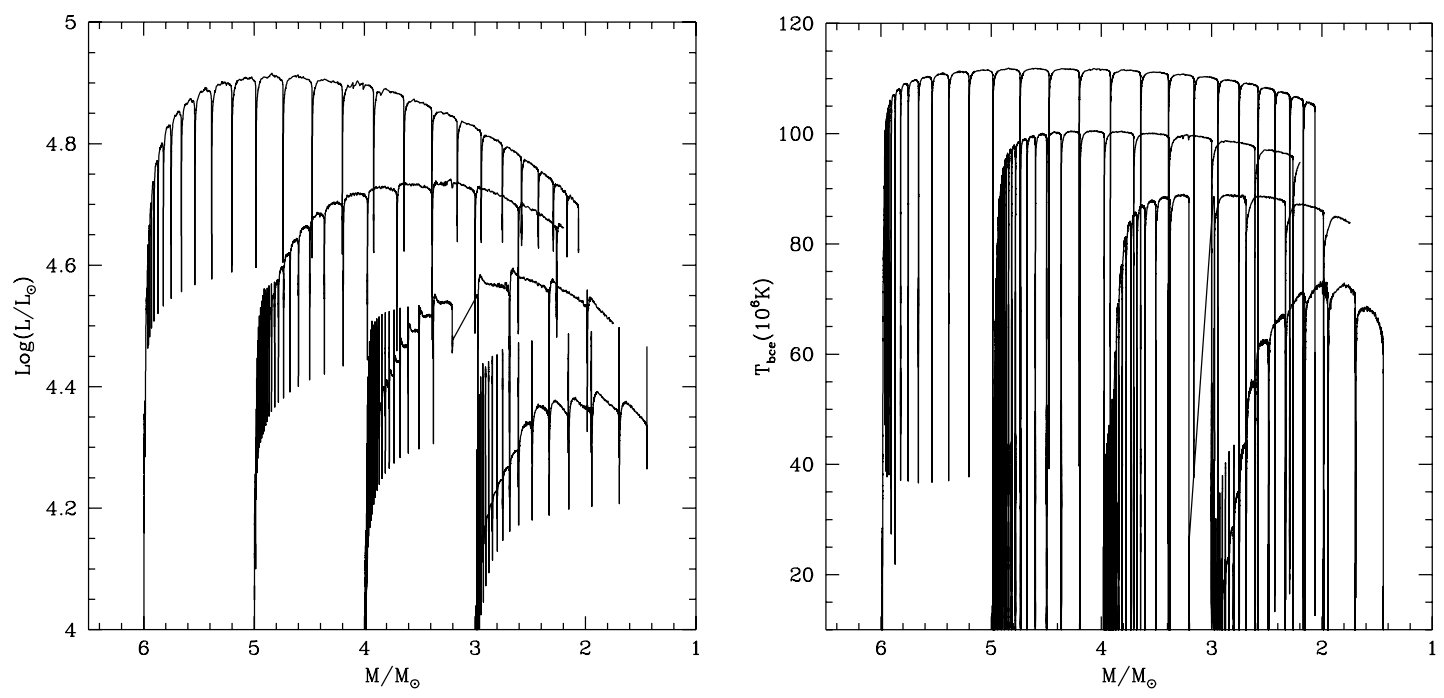

Fig. 1. Variation with the total mass of the luminosity (left) and temperature at the bottom of the convective envelope (right) of some intermediatemass models during the AGB phase. For clarity, only models corresponding to initial masses $3,4,5,6 M_{\odot}$ are shown.

Table 2. Chemical composition of the ejecta of intermediate-mass models.

\begin{tabular}{cccccccccccc}
\hline \hline$M / M_{\odot}$ & $\eta_{\mathrm{R}}$ & $Y$ & $A(\mathrm{Li})$ & {$\left[{ }^{12} \mathrm{C} / \mathrm{Fe}\right]$} & {$\left[{ }^{14} \mathrm{~N} / \mathrm{Fe}\right]$} & {$\left[{ }^{16} \mathrm{O} / \mathrm{Fe}\right]$} & {$\left[{ }^{19} \mathrm{~F} / \mathrm{Fe}\right]$} & {$\left[{ }^{23} \mathrm{Na} / \mathrm{Fe}\right]$} & {$[\mathrm{Mg} / \mathrm{Fe}]$} & {$\left[{ }^{27} \mathrm{Al} / \mathrm{Fe}\right]$} & $R(\mathrm{CNO})$ \\
\hline 3.0 & 0.02 & .248 & 2.77 & 0.84 & 2.21 & 0.92 & 0.10 & 1.16 & 0.57 & 0.65 & 9.6 \\
3.5 & 0.02 & .265 & 2.43 & 0.51 & 2.18 & 0.77 & -0.26 & 1.30 & 0.55 & 0.66 & 7.9 \\
4.0 & 0.02 & .281 & 2.20 & 0.14 & 2.02 & 0.44 & -0.61 & 1.18 & 0.48 & 0.55 & 4.9 \\
4.5 & 0.02 & .310 & 2.00 & 0.12 & 1.89 & 0.19 & -0.90 & 0.97 & 0.43 & 0.85 & 3.1 \\
5.0 & 0.02 & .324 & 1.98 & 0.13 & 1.70 & -0.06 & -1.16 & 0.60 & 0.35 & 1.02 & 2.1 \\
5.5 & 0.02 & .334 & 1.93 & -0.41 & 1.51 & -0.35 & -1.39 & 0.37 & 0.28 & 1.10 & 1.3 \\
6.0 & 0.02 & .343 & 2.02 & -0.62 & 1.35 & -0.40 & -1.36 & 0.31 & 0.29 & 1.04 & 0.97 \\
6.3 & 0.02 & .348 & 2.06 & -0.68 & 1.33 & -0.37 & -1.28 & 0.30 & 0.30 & 0.99 & 0.94 \\
5.0 & 0.01 & .327 & 1.79 & 0.00 & 1.83 & -0.14 & -1.30 & 0.67 & 0.40 & 1.20 & 2.77 \\
5.0 & 0.04 & .323 & 2.49 & -0.37 & 1.58 & -0.05 & -1.00 & 0.70 & 0.39 & 0.80 & 1.71 \\
6.0 & 0.01 & .345 & 1.83 & -0.40 & 1.49 & -0.42 & -1.45 & 0.27 & 0.22 & 1.07 & 1.29 \\
\hline
\end{tabular}

as $[\mathrm{A} / \mathrm{Fe}]=\log (\mathrm{A} / \mathrm{Fe})_{\text {ejecta }}-\log (\mathrm{A} / \mathrm{Fe})_{\odot}$, while the abundances are mass fractions. The only exceptions are helium and lithium for which we respectively list the mass fraction $Y$ and the standard $A(\mathrm{Li})$ quantity. Note that $\mathrm{Mg}$ in Col. 9 refers to the total magnesium abundance, and $R(\mathrm{CNO})$ in the last column gives the ratio between the total $\mathrm{C}+\mathrm{N}+\mathrm{O}$ in the ejecta and the initial value.

\subsection{The oxygen depletion}

The depletion of the surface oxygen abundance requires a strong $\mathrm{HBB}$ at the bottom of the convective envelope, as the activation of the full CNO cycle demands temperatures approaching $100 \mathrm{MK}$, which, within the context of the AGB modelling, are only attainable via a very efficient description of convection (Ventura \& D'Antona 2005b). The present models were calculated with the FST prescription; thus, at least in the most massive models, we expect to reach such a high $T_{\text {bce, }}$ as can be seen in the right panel of Fig. 1 .

The left panel of Fig. 2 shows the variation with the total mass of the surface oxygen abundance for the masses examined here, with the only exception of the $6.3 M_{\odot}$ model, which was omitted for the sake of clarity, because it is very similar to the $6 M_{\odot}$ case. We see that oxygen burning starts efficiently shortly after the beginning of the AGB phase in all the models that are more massive than $4 M_{\odot}$. In the same panel we easily see the effects of the TDU, which increases the oxygen content of the envelope when efficient, because carbon- and oxygen-rich material is dredged up from the ashes of the preceding $3 \alpha$-burning shell that forms during each TP. A strong oxygen depletion is thus inhibited by repeated and efficient TDU episodes, so that in the less massive models, which undergo many TDUs and hardly reach the temperatures requested to ignite oxygen burning, the oxygen is indeed produced rather than destroyed within their envelopes. With increasing mass, we shift progressively to a situation where the final oxygen abundance is a delicate compromise between the depletion triggered by the activation of the full CNO cycle and the increase due to the TDU $\left(4.5 M_{\odot} \leq M \leq 5.5 M_{\odot}\right)$, to end up with the most massive models $\left(M \geq 6 M_{\odot}\right)$. In these oxygen can be eventually depleted by a factor $\sim 20$ compared to the initial value (see the track corresponding to the $6 M_{\odot}$ model in the left panel of Fig. 2).

In the right panel of Fig. 2 we show the oxygen content of the ejecta of the models calculated, in terms of $[\mathrm{O} / \mathrm{Fe}]$, to allow a more straight comparison with the observations outlined in Sect. 2. We note a very high oxygen content in the ejecta of the models with masses $M<4 M_{\odot}$, consistent with the previous discussion, the model with initial mass $4 M_{\odot}$ shows an unchanged oxygen content compared to the initial $\alpha$-enhanced value. The mass-oxygen trend is progressively decreasing with mass and reaches a plateau value of $[\mathrm{O} / \mathrm{Fe}] \sim-0.4$ dex for all the masses $M>5 M_{\odot}$. The reasons this lower limit exists are twofold: a) on the one hand (see Col. 8 in Table 1), we saw that there is an upper limit for $T_{\text {bce }}$, which therefore limits the degree of O-burning 

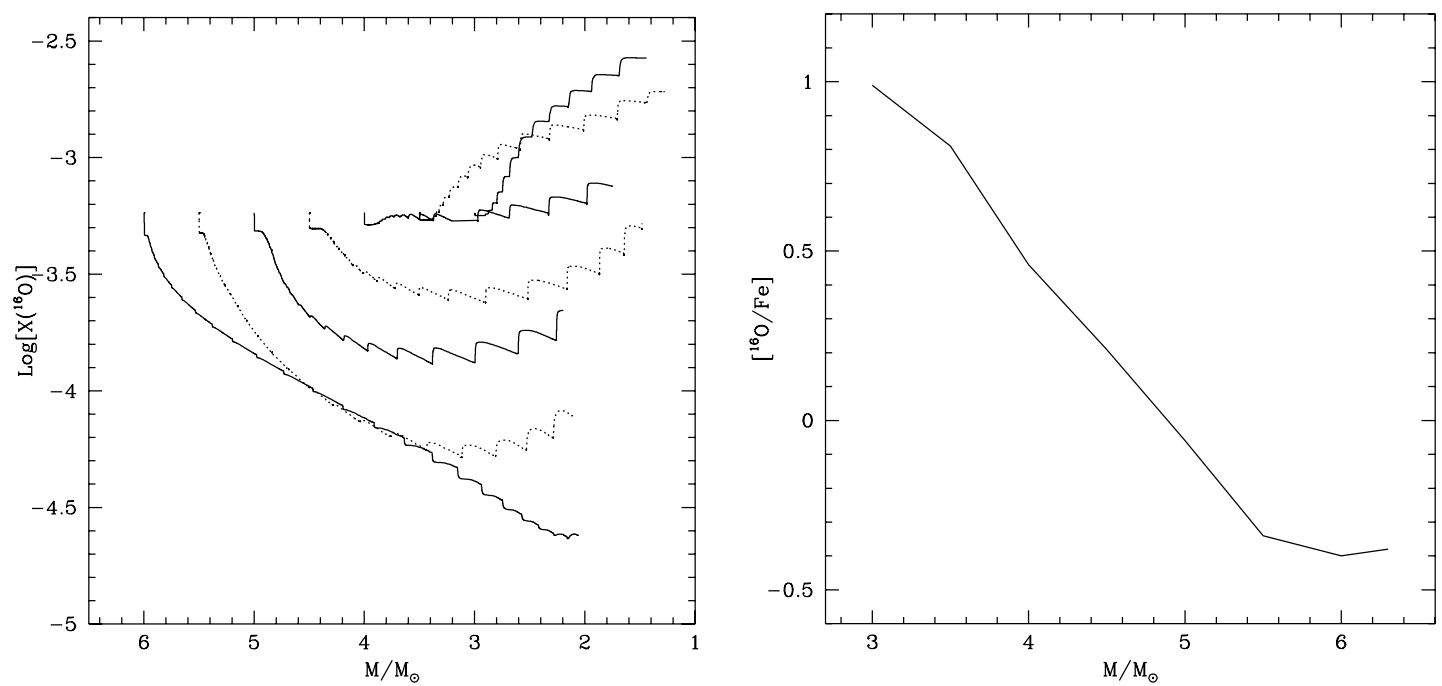

Fig. 2. Left: variation with the total mass of the surface oxygen mass fraction of the AGB models with masses $3 M_{\odot} \leq M \leq 6 M_{\odot}$; the strong depletion in the most massive models is a clear signature of strong HBB at the bottom of the convective envelope. Right: oxygen content of the ejecta as a function of the initial mass; a plateau value of $[\mathrm{O} / \mathrm{Fe}]=-0.4$ is reached for the highest masses

that may be achieved at the bottom of the envelope; b) the luminosity in the most massive models is so high that they lose mass rapidly already from the very first TPs, when the oxygen abundance is still high (see the different slope of the oxygen-mass relation characterising the $6 M_{\odot}$ model compared to the other masses in the left panel of Fig. 2). We will show that changing the mass-loss description does not substantially change this conclusion. Finally, we note that this lower limit for $[\mathrm{O} / \mathrm{Fe}]$ is in good agreement with the lowest oxygen abundances measured in TO and SGB stars belonging to intermediate metallicity GCs.

\section{2. aluminium production and the activation of the Mg-Al cycle}

Columns 10 and 11 of Table 2 report the $\mathrm{Mg}$ and $\mathrm{Al}$ content of the ejecta. While the observed spread in the Mg abundance, as outlined in Sect. 3, is still debated and, in any case, restricted to $0.2-0.4 \mathrm{dex}$, the stars in GCs showing the highest degree of oxygen depletion are also strongly enriched in aluminium, with $[\mathrm{Al} / \mathrm{Fe}]=1$ in the most extreme cases (Gratton et al. 2001; Sneden et al. 2004).

In the AGB modelling, an aluminium production at the bottom of the envelope is made possible by the activation of the $\mathrm{Mg}-\mathrm{Al}$ chain, which, favouring proton captures by the heavy isotopes of magnesium, eventually leads to the synthesis of ${ }^{27} \mathrm{Al}$ (Denissenkov \& Herwig 2003; Ventura \& D'Antona 2005a). Also, TDU plays a role that is not negligible, as a deep penetration of the convective envelope within the region previously touched by helium burning may bring ${ }^{25} \mathrm{Mg}$ and ${ }^{26} \mathrm{Mg}$ synthetised via $\alpha$-captures by ${ }^{22} \mathrm{Ne}$ nuclei to the surface. These isotopes, once the CNO-burning shell is reactivated, undergo proton capture and produce aluminium.

Figure 3 shows the evolution of the surface aluminium content in the models presented here. We note in all cases a trend to increase during the evolution; in the most massive models, due to the stronger nucleosynthesis activated, the aluminium production is greater. Even in this case, as for the depletion of oxygen, we note an upper limit to the aluminium enhancement, which can also be seen in Col. 10 of Table 2 to be $[\mathrm{Al} / \mathrm{Fe}]=1$. The reasons for the impossibility of a higher Al production are the upper limit of $T_{\text {bce }}=110 \mathrm{MK}$ found in our models, the negligible

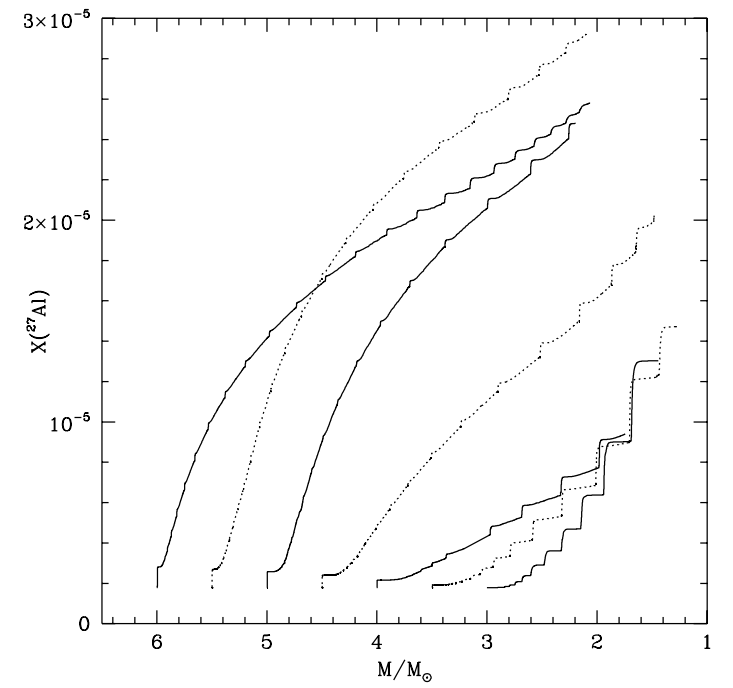

Fig. 3. The variation during the AGB phase of the surface aluminium abundance, for the same models reported in Fig. 2. Note the combined effects of HBB and of TDU to increase the surface aluminium content

extent of TDU in the most massive stars, preventing the transport of the magnesium isotopes from the interior to the surface, and the strong mass loss suffered from these models at the beginning of the AGB phase, which favours the ejection into the ISM of material that is not aluminium-rich.

We see from Col. 11 of Table 2 that the ejecta of all the models are characterised by $0.5 \leq[\mathrm{Al} / \mathrm{Fe}] \leq 1$ and are therefore fully consistent with the aluminium content of the stars with the anomalous chemistry outlined by the spectrospic investigations presented in Sect. 3.

The total magnesium abundance, as is evident from the previous discussion, is the result of the balance between the increase in $\mathrm{Mg}$ determined by the TDU and the depletion due to proton captures during HBB. This explains the negative trend with mass that can be seen in Col. 9 of Table 2 .

We underline here the striking difference between our findings and the results obtained by Fenner et al. (2004, see the bottom panel of their Fig. 1), where they find that the most Al-rich 
stars were also magnesium-rich. This was a result of the effects of many TDUs, enriching the envelope with the heavy magnesium isotopes produced in the $3 \alpha$ shell. The different treatment of convection between the two sets of models leads to this discrepancy, because the use of the FST model reduces the number of TPs and TDUs.

In contrast to oxygen, the predictive power of our results for $\mathrm{Al}$ is undermined by the range of uncertainties related to the relevant cross-sections. Izzard et al. (2007) show that in massive AGBs the yields of ${ }^{27} \mathrm{Al}$ is affected by the uncertainties connected to both the ${ }^{26} \mathrm{Mg}(\mathrm{p}, \gamma){ }^{27} \mathrm{Al}$ and ${ }^{26} \mathrm{Al}(\mathrm{p}, \gamma){ }^{27} \mathrm{Si}$ reaction rates. We used the upper NACRE limit for these reactions in the present investigation, but we keep in mind that these results have an associated uncertainty that may be estimated to be around $0.3-0.4$ dex.

\subsection{The puzzling behaviour of sodium}

The debate on the amount of sodium that may be synthesised within AGBs is still open, due to the large uncertainties associated to a) the cross-sections of the reactions involved in the $\mathrm{Ne}-\mathrm{Na}$ cycle, b) the cross sections of the $\alpha$-captures by ${ }^{22} \mathrm{Ne}$, and c) other physical inputs that play a role in determining the sodium content within the envelope of these stars.

As outlined by Ventura \& D'Antona (2006), the surface sodium abundance first increases due to the II DUP, then, particularly in AGB models calculated with an efficient convective model, it is further produced by burning of the dredged-up ${ }^{22} \mathrm{Ne}$, and is later decreased when the rate of destruction exceeds that of production. Any TDU favours sodium production, due to dredging up of primary ${ }^{22} \mathrm{Ne}$ synthesised via $\alpha$-capture within the convective shell that forms during the TP. This behaviour is confirmed by Fig. 4, that shows the variation in the surface sodium abundance of the evolutionary models. The behaviour of the $M \leq 4 M_{\odot}$ stars qualitatively agrees with the AGB models used by Fenner et al. (2004, see the upper panel of their Fig. 1): we note a large increase in the sodium abundance, due to the dredge-up of ${ }^{22} \mathrm{Ne}$, which is later converted to sodium. Contrary to their findings, our more massive models show an opposite behaviour, because the FST convective model favours a more advanced nucleosynthesis, with a partial destruction of the sodium created before. We also recall that the higher mass loss favours a smaller number of TPs, thus acting against sodium production.

The precedent discussion explains the clearly negative trend with mass of the $[\mathrm{Na} / \mathrm{Fe}]$ values in the 9 th column of Table 2. We focus our attention on the most massive models, which produces yields we saw to be aluminium-rich and oxygen-poor. The sodium content of their ejecta is in the range $0.3-0.4 \mathrm{dex}$, which is consistent with the sodium abundances derived by most of the research groups for the stars with the most anomalous chemical composition.

The uncertainties connected to the cross sections have a dramatic impact on the value of $[\mathrm{Na} / \mathrm{Fe}]$ of the ejecta, even more than we saw for aluminium. Concerning HBB, the main problems are associated to the cross section of the ${ }^{22} \mathrm{Ne}(\mathrm{p}, \gamma)^{23} \mathrm{Na}$ reaction, which is uncertain by a factor 2000 (Hale et al. 2002); even the other reaction related to determining the correct sodium equilibrium value (i.e. ${ }^{23} \mathrm{Na}(\mathrm{p}, \alpha)^{20} \mathrm{Ne}$ ) has a margin of uncertainty that is however smaller ( 30\%; Hale et al. 2004).

We ran three models with initial masses $4,5,6 M_{\odot}$, where we used the lower limit for the ${ }^{22} \mathrm{Ne}(\mathrm{p}, \gamma)^{23} \mathrm{Na}$ reaction and the upper limit for ${ }^{23} \mathrm{Na}(\mathrm{p}, \alpha)^{20} \mathrm{Ne}$. The comparison between these simulations (dotted tracks) and those described in Table 2 (solid) is shown in Fig. 5. We note that when the cross sections minimising

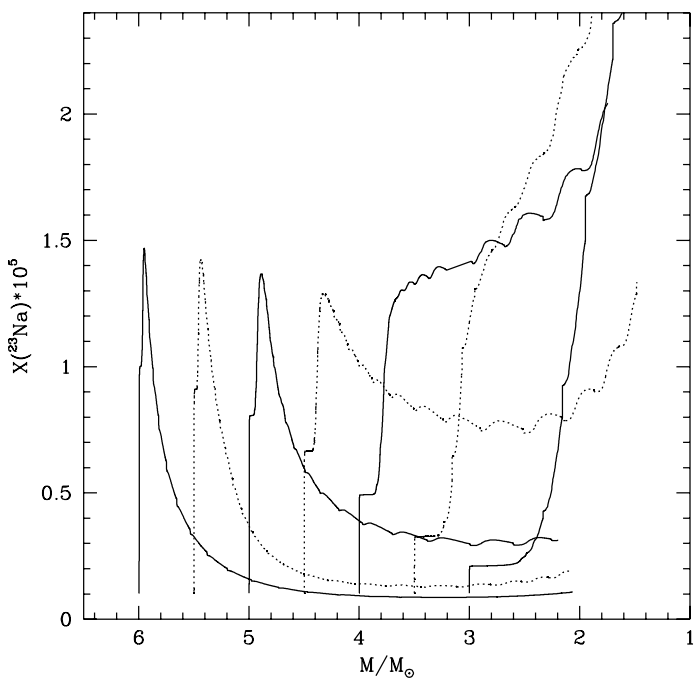

Fig. 4. Variation with mass of the surface sodium abundance within our standard models.

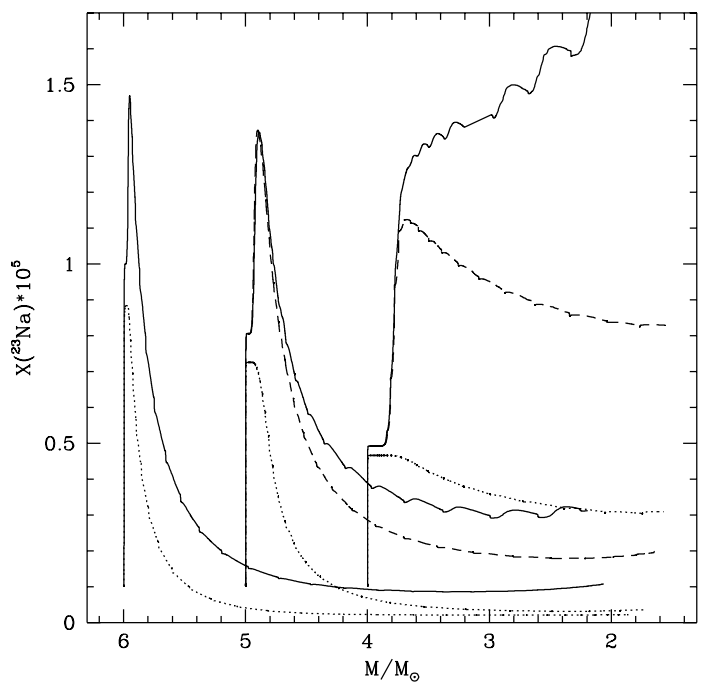

Fig. 5. Variation of the sodium surface content within models with initial masses 4, 5, $6 M_{\odot}$ calculated with the recommended values of the cross sections of the Ne-Na cycle (dotted) and those maximising sodium production (see text for details). The dashed tracks show the results obtained when the upper limits of the $\alpha$-capture reactions by ${ }^{22} \mathrm{Ne}$ nuclei are adopted.

the sodium production are used, with the only exception of the II DUP, sodium is destroyed when $T_{\text {bce }}$ becomes large enough to ignite proton capture by ${ }^{23} \mathrm{Na}$ nuclei. The sodium contents that we get are considerably lower; i.e. $[\mathrm{Na} / \mathrm{Fe}]=0.6$ for the $4 M_{\odot}$ model, $[\mathrm{Na} / \mathrm{Fe}]=0.0$ in the $M=5 M_{\odot}$ case, and $[\mathrm{Na} / \mathrm{Fe}]=-0.2$ for $M=6 M_{\odot}$. These values are lower than those reported in Table 2 by 0.6 dex. Note that this is not proportional to the reduction factor of the ${ }^{22} \mathrm{Ne}(\mathrm{p}, \gamma)^{23} \mathrm{Na}$ reaction, because, as pointed out by Izzard et al. (2007), once ${ }^{22} \mathrm{Ne}$ is destroyed at the bottom of the envelope, no more sodium can be created, despite the use of a cross section for ${ }^{22} \mathrm{Ne}$ burning, which is a factor 2000 higher. This is also consistent with the investigation by Ventura \& D'Antona (2006), who point out that a decrease in the ${ }^{23} \mathrm{Na}(\mathrm{p}, \alpha)^{20} \mathrm{Ne}$ reaction by a factor 2 could better reconcile the sodium content of the ejecta of the most massive AGBs of intermediate metallicity with the spectrospic measurements of GC stars. 

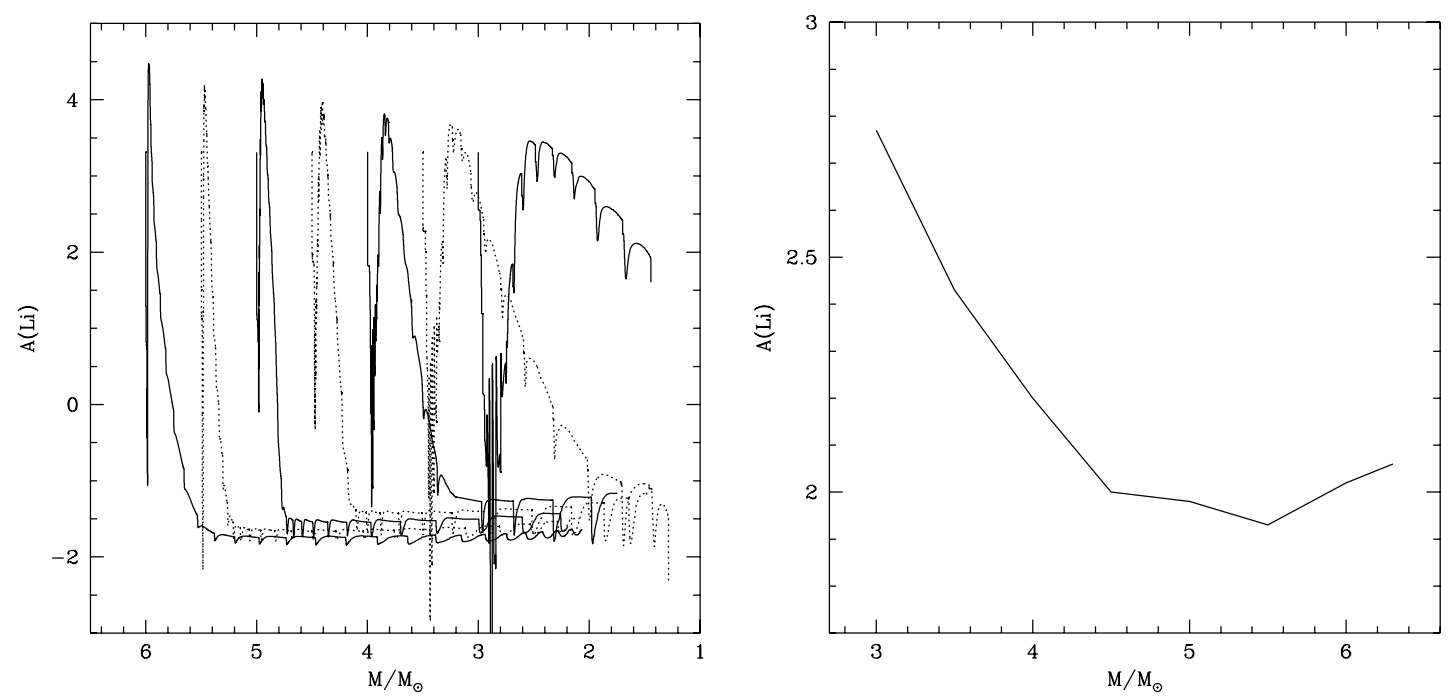

Fig. 6. Left: variation as a function of the total stellar mass of the surface lithium content of the intermediate mass models during the whole AGB evolution. Right: the lithium content of the ejecta as a function of the initial mass.

For the models experiencing TDU, another source of uncertainty for the sodium yield is provided by the cross sections of the $\alpha$-capture reactions by ${ }^{22} \mathrm{Ne}$ nuclei, whose upper limit is 3 orders of magnitude higher than the recommended values (Angulo et al. 1999). These reactions determine the ${ }^{22} \mathrm{Ne}$ content in the convective shell that forms during the TP; hence the amount of ${ }^{22} \mathrm{Ne}$ that may be dredged-up in the after-pulse phase, and therefore the quantity of sodium that may be synthesised at the bottom of the envelope via proton capture by ${ }^{22} \mathrm{Ne}$ nuclei.

The results obtained when these upper limits are used are shown in Fig. 5: for the 4 and $5 M_{\odot}$, the overall sodium abundance is clearly reduced. Unlike the previous case, the associated uncertainties on the sodium yields are not uniform with mass, but instead show a decreasing trend, ranging from no effect for the most massive models, to $\delta[\mathrm{Na} / \mathrm{Fe}] \sim 0.15$ dex for $M=5 M_{\odot}$, up to $\delta[\mathrm{Na} / \mathrm{Fe}] \sim 0.3$ dex for $M=4 M_{\odot}$. These results are actually not surprising, since dredging up of ${ }^{22} \mathrm{Ne}$ becomes progressively greater in determining the sodium abundance the lower is the stellar mass.

The yields of sodium from these sources are still highly uncertain. Only a more solid estimate of the relevant cross-sections may help increase the reliability of these investigations.

\subsection{The lithium problem}

Lithium is synthesised at the bottom of the convective envelope of AGBs at temperatures exceeding $40 \times 10^{6} \mathrm{~K}$ via the Cameron \& Fowler (1971) mechanism. Sackmann \& Boothroyd (1992) first showed that the use of a diffusive approach was mandatory to describe such a delicate interplay among nuclear and mixing time scales, which could eventually lead to lithium production. Ventura et al. (2000) used this approach to reproduce the lithium vs. luminosity trend observed in the Large Magellanic Cloud.

The key factor for achieving lithium production is the activation of the ${ }^{3} \mathrm{He}(\alpha, \gamma)^{7} \mathrm{Be}$ reaction at the bottom of the convective envelope, which is possible when $T_{\text {bce }}>40 \times 10^{6} \mathrm{~K}$.

The left panel of Fig. 6 shows that all our models attain temperatures at the bottom of the convective envelope that are high enough to ignite the Cameron \& Fowler mechanism. The most massive stars undergo a rapid consumpion of the whole ${ }^{3} \mathrm{He}$ available in the envelope, so that the surface lithium content rapidly declines to extremely low abundances after reaching a maximum value $A(\mathrm{Li}) \sim 4$. This process becomes progressively slower as the mass decreases, so we end up with the $3 M_{\odot}$ model, which is still lithium-rich at the end of its AGB evolution. This discussion explains the relation between the initial mass of the star and the lithium content of its ejecta, shown in the right panel of Fig. 6. All the massive models have $A(\mathrm{Li}) \sim 2$, in excellent agreement with the lithium abundance of the oxygen-poor TO stars in NGC 6752 (Pasquini et al. 2005).

\subsection{The helium enrichment}

The AGB models discussed here experience a small number of TPs, thus most of the helium enrichment of the envelope takes place during the II DUP. Since this is deeper the higher the initial mass of the star (see Col. 5 of Table 1), we expect the helium enrichment to increase with mass, as is confirmed by the results reported in Col. 3 of Table 2. We find a small increase (compared to the standard Big Bang value, $Y=0.24$ ) in the models with mass $M<4 M_{\odot}$, and the maximum enrichment, for the masses close to the limit for carbon ignition, is $Y=0.35$. We will discuss in a forthcoming paper (Pumo et al. 2007) the overall helium enrichment due to AGB and super-AGB stars of intermediate metallicity, and the robustness of these predictions, related essentially to the efficiency of the II DUP.

\subsection{Comparison with previous models}

Ventura et al. (2002) present models for the evolution of stars with intermediate mass at various metallicities, ranging from $Z=2 \times 10^{-4}$ to $Z=0.01$. We compare the yields of the present work with the results of that investigation for the metallicity $Z=0.001$. First, we note that the helium content is systematically higher here by $\sim 0.04$, as can be seen by comparing the third column of Table 2 with the dashed curve giving the heliummass trend in Fig. 4 in Ventura et al. (2002). These results may be understood in terms of the extra-mixing from the bottom of the convective envelope that was assumed in the present models and that was neglected in Ventura et al. (2002).

The combined effects of the overshooting from the envelope and the different mixture adopted (solar in Ventura et al. (2002), $\alpha$-enhanced here) favours lower core masses in the present 


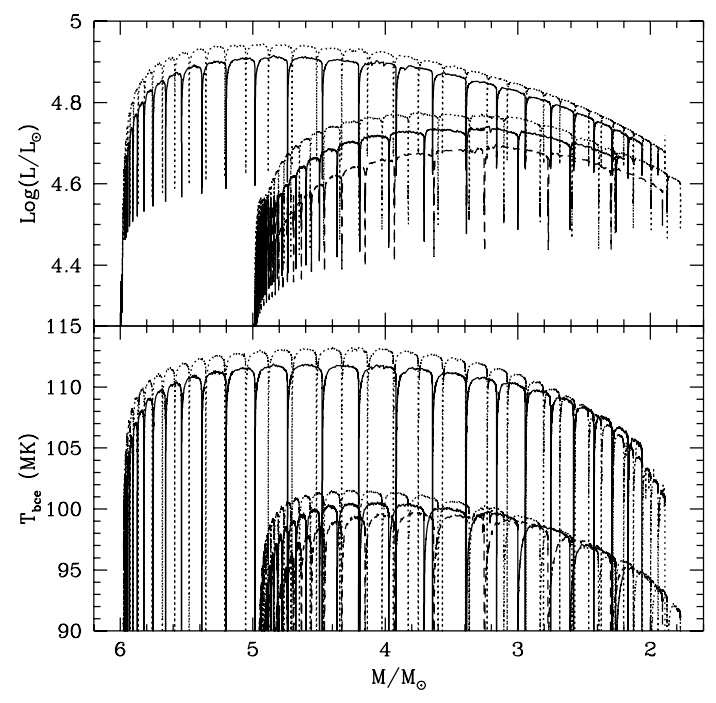

Fig. 7. Comparison of the variation in the total mass of the luminosity (top) and temperature at the bottom of the convective envelope (bottom) of models with initial masses $5,6 M_{\odot}$, calculated with different values of the parameter $\eta_{\mathrm{R}}$ enetering Eq. (2).

models, so that a given model here can be compared with a model $0.5 M_{\odot}$ less massive in Ventura et al. (2002).

Even with this assumption, we note differences in the CNO yields, the overall $\mathrm{C}+\mathrm{N}+\mathrm{O}$ contribution being higher here compared to the corresponding values in Ventura et al. (2002); see Fig. 6 in Ventura et al. (2002), to be compared with Cols. 5 to 7 in Table 2. The reason for such a discrepancy is the different efficiency of the TDU found in the two sets of models: we find a maximum efficiency of $\lambda=0.7$ here (which decreases to $\lambda=0.5$ in the more massive models), whereas $\lambda$ could hardly reach 0.5 in Ventura et al. (2002). This change, favoured by the different mixture adopted, determines the differences in the yields obtained.

The detailed comparison of the other yields is difficult because of the differences in the nuclear cross-sections adopted for the relevant reactions. Ventura et al. (2002) used the NACRE compilation, whereas here we use the most updated releases in the literature.

\section{The uncertainties related to mass loss}

Mass loss plays a fundamental role in the context of AGB evolution. It is the reduction of the convective envelope via stellar winds that eventually halts the TP phase and leads to PN ejection. Also, the description of mass loss determines the number of TPs experienced by the star and, in turn, the number of TDUs (Schönberner 1979).

Ventura et al. (2000) calibrated the parameter $\eta_{\mathrm{R}}$ entering Eq. (2) by reproducing the luminosity function of lithium-rich stars in the Magellanic Clouds. The chemistry of the stars examined in the present work is different, thus leaving some room for a possible variation in $\eta_{\mathrm{R}}$. It is therefore essential to understand to what extent the yields we obtain depend on this choice.

On the physical side, a greater mass loss leads the star to evolve at lower luminosities; the degree of the nucleosynthesis achieved at the bottom of the convective envelope is reduced, because we have lower temperatures. This is confirmed by Fig. 7, where we show the evolution of the luminosity and of $T_{\text {bce }}$ in models with initial masses 5 and $6 M_{\odot}$, calculated with different $\eta_{\mathrm{R}} \mathrm{s}$.
Chemically, the situation is complex, and not all the isotopes follow the same trend with $\dot{M}$. This is dependent on the modality with which any chemical species is synthesised (or destroyed) within the convective envelope. The last three lines of Table 2 report the chemistry of the ejecta of the models of 5 and $6 M_{\odot}$ calculated with a different mass-loss rates with respect to our standard case.

The behaviour of lithium is the most linear. As can be seen in the left panel of Fig. 8, lithium is produced only during the first TPs, so that the average lithium content of the ejecta is essentially determined by the mass lost by the star during this phase. A large $\dot{M}$ allows a larger release of lithium-rich material during the early phases of the AGB evolution, with a consequent increase in $A(\mathrm{Li})$. For a given mass, we see in Table 2 an almost linear trend $A(\mathrm{Li})-\eta_{\mathrm{R}}$. A similar straight path is also followed by fluorine, which is destroyed during the very first TPs, so that the overall yield is determined by the strength of the stellar winds during the first TPs. In this case the slope of the $[\mathrm{F} / \mathrm{Fe}]-\eta_{\mathrm{R}}$ relation is flatter compared to lithium, because the fluorine consumption is faster than the duration of the whole phase of lithium production and destruction.

Other elements show up a less defined behaviour, because their yield is determined by the nucleosynthesis at the bottom of the envelope during the whole AGB phase, and also by the TDU. Interestingly, we find that in some cases the yields are not very sensitive to the mass-loss rate adopted. A typical example is oxygen, for which we show the variation in the surface abundance in the right panel of Fig. 8. When mass loss is reduced, the tendency of the oxygen abundance of the ejecta to diminish (less oxygen-rich matter during the first TPs is lost by the star) is partly compensated for by the larger number of TPs, which act to increase the surface oxygen content. The clearest example is the $6 M_{\odot}$ model, for which a smaller $\eta_{\mathrm{R}}$ (dotted track) leads to a smaller surface oxygen abundance until the total mass of the star drops to $3 M_{\odot}$, but to a larger mass fraction in the latest evolutionary phases, when the TDU becomes efficient. As can be seen in Table 2, the oxygen content of the ejecta is almost independent of $\eta_{\mathrm{R}}$, and so is the general conclusion that we reached in Sect. 5.1 concerning the maximum depletion of oxygen obtainable by these models. Playing with mass loss leaves unchanged the maximum depletion of oxygen obtainable at these metallicites, leading to a minimum oxygen content of the ejecta $[\mathrm{O} / \mathrm{Fe}]=-0.4$.

A behaviour similar to oxygen is also followed by sodium, again because its abundance in the envelope is a balance between destruction via proton capture and production via dredging up of ${ }^{22} \mathrm{Ne}$. Independent of mass, the related uncertainty is $\delta[\mathrm{Na} / \mathrm{Fe}] \sim 0.1$ dex.

The aluminium abundance of the ejecta decreases with $\dot{M}$, because a higher mass loss favours a larger release of mass at the beginning of the AGB phase, when the aluminium has not yet been synthesised in great amounts. The smaller number of TPs at large $\dot{M}$ tends to increase the ${ }^{27} \mathrm{Al}$ abundance indirectly, via dredge-up of the heavy magnesium isotopes, which later form aluminium via proton capture. The variation in $[\mathrm{Al} / \mathrm{Fe}]$ with the mass loss rate is $(\delta[\mathrm{Al} / \mathrm{Fe}] \sim 0.1 \mathrm{dex})$.

Helium is not influenced by the details of the mass-loss description, because the only substantial change in its surface abundance takes place during the II DUP and remains approximately constant during the whole subsequent AGB phase.

Finally, we note from the last column of Table 2 that the $\mathrm{CNO}$ ratio has a negative trend with mass loss, which is a mere consequence of the fact that, when $\dot{M}$ is high, the star experiences a smaller number of TPs. 

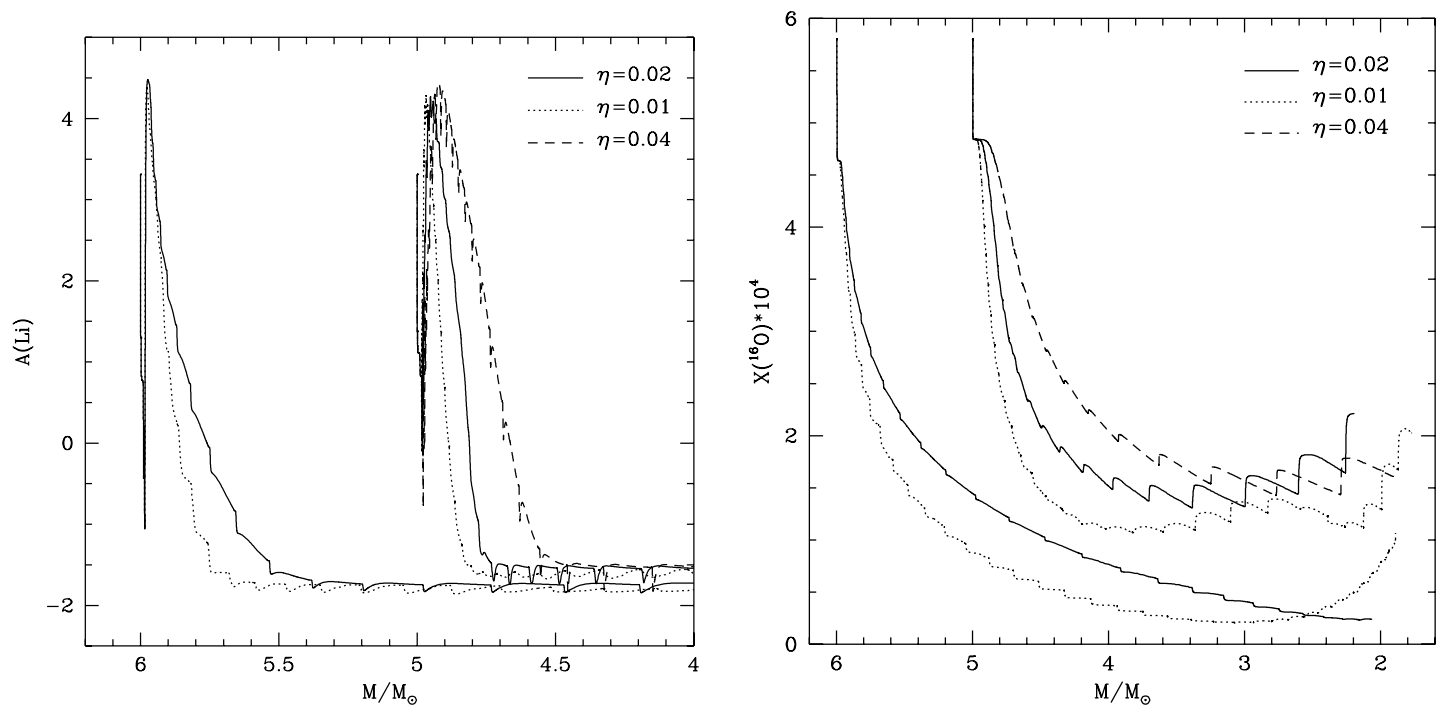

Fig. 8. Variation with the total mass of the star of the lithium (left) and oxygen (right) surface abundances within models of initial mass 5 and $6 M_{\odot}$ calculated with different mass loss rates. Note the straightforward dependency of lithium on mass loss, compared to the more tricky behaviour of oxygen, whose destruction is first amplified by a lower mass loss rate, and later prevented by TDU episodes.

We may summarise the effects of the uncertainties of mass loss during the AGB phase on the chemistry of the ejecta as follows:

1. oxygen is not sensitive to the mass loss rate adopted;

2. sodium and aluminium show a positive trend with mass loss, but the uncertainty associated with the adopted description of mass loss is considerably smaller than the indetermination due to the unknown relevant cross-sections;

3. lithium and fluorine are more sensitive to mass loss, with a linear positive trend;

4. the CNO content of the ejecta is sensitive to mass loss, because a stronger $\dot{M}$ diminishes the number of TPs experienced by the stars, thus favouring a lower $\mathrm{C}+\mathrm{N}+\mathrm{O}$ abundance.

\section{Discussion: theory vs. observations}

We discuss the self-enrichment scenario hypothesis for intermediate metallicity clusters, by asking whether the ejecta of the most massive AGBs can account for the chemical patterns traced by the abundances of the GC stars with the most anomalous chemistry. We restrict our attention to the least evolved stars (i.e. TO and SGB sources, or giants well below the RGB bump), despite the difficulties presented by their spectroscpic analysis, because this allows us to rule out any possible change in the surface chemistry due to some non-canonical extra mixing while ascending the RGB. We therefore disentangle the primordial from the evolutionary effects and focus only on the abundance patterns present directly in the matter from which the stars formed.

Our goal is to test the possibility that our ejecta can reproduce the observed $\mathrm{O}-\mathrm{Na}$ and $\mathrm{O}-\mathrm{Al}$ trends and that the stars showing the strongest oxygen depletion are fluorine poor and possibly sligthly depleted in magnesium. Finally, we compare our results with the recent analysis of the lithium abundances in NGC 6752, which indicate that oxygen-poor stars deviate from the Spite's plateau, having a lithium content a factor 2 smaller than the standard value (Pasquini et al. 2005).

In making this comparison, we keep in mind that the oxygen abundances predictions are very robust, that the aluminium and even more sodium mass fractions are made uncertain by the poor knowledge of the relevant cross-sections in the range of temperatures of interest here $(T \sim 100 \mathrm{MK})$, and that lithium is strongly influenced by the assumed mass-loss rate at the beginning of the AGB phase.

The left panel of Fig. 9 shows the observed oxygen-sodium trend for such stars. According to our interpretation, the observed points inside the squared box in the right lower portion of the plane represent stars born with the original chemistry, while those belonging to the 2nd generation, whose initial chemistry traces the pollution by AGBs, are included within the squared box in the left-upper part. The remaining points, with high sodium and normal oxygen, may be stars formed by processed matter mixed with remnant primordial gas (Decressin et al. 2007). The uncertainties related to the cross-sections strongly limit the predictive power of the results obtained, and the observational spread $(\delta[\mathrm{Na} / \mathrm{Fe}] \sim 0.3$ for a given $[\mathrm{O} / \mathrm{Fe}])$ complicates the comparison. However, we note that the ejecta of models with masses $M \geq 5 M_{\odot}$ might account for the oxygen and sodium abundances detected in the 2 nd generation of stars. Notice that here we are again touching the problem of the mass budget. If only the envelopes of stars from 5 up to $6.3 M_{\odot}-$ or at most up to $7-8 M_{\odot}$, if we can assume that the superAGBs also contribute with similar yields - can form the self-enriched stars, the gas contained in this mass range, for reasonable IMFs, is only a few percent of the total initial cluster mass. As we note in the introduction, we must then hypothesise that the initial cluster mass was much higher than the present mass and that the second generation stars have been lost during the long-term cluster evolution.

The right panel of Fig. 9 reports the observed points in the $\mathrm{O}-\mathrm{Al}$ plane. Even in this case we report two theoretical lines, obtained with different choices for the rates of the $\mathrm{Mg}-\mathrm{Al}$ chain reactions. In this case the observed trend is clearly reproduced, in particular for the chemistry of the most oxygen-poor stars, which show an aluminium enhancement by $[\mathrm{Al} / \mathrm{Fe}] \sim 1$. In the case of aluminium the comparison is more straightforward, because the theoretical uncertainties related to the cross-sections are lower. The two squares in the figure have the same meaning as in the left panel. 

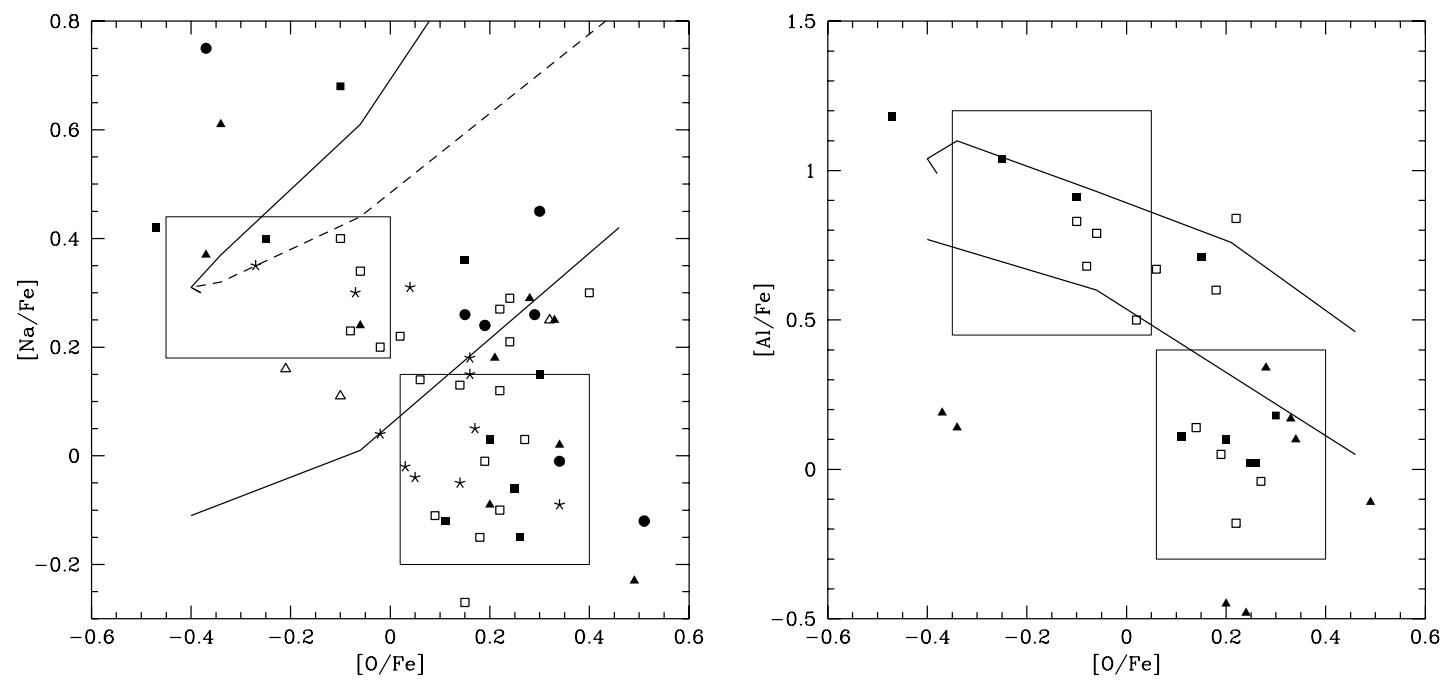

Fig. 9. Left: the observed oxygen-sodium trend in stars in GCs. The solid lines indicate the content of the ejecta of our models, obtained with the recommended cross-sections for the reactions involved in the Ne-Na cycle (lower track), and with the reactions rates maximizing sodium production (upper lines). The dashed track shows the abundances of the ejecta from the models calculated with the enhanced cross sections for the $\alpha$-captures by ${ }^{22} \mathrm{Ne}$. Right: the observed oxygen-aluminium anticorrelation. The two solid lines indicate the results from our models, according to the choices made for the cross-section of the Mg-Al reactions. The observed points refer to the following works. Full triangles: NGC 6752 (Gratton et al. 2001); open squares: M 5 stars with $V>16$ (Ramirez \& Cohen 2003); stars: M 13 stars with $V>15$ (Cohen \& Melendez 2005); open triangles: M3 stars with $V>15$ (Cohen \& Melendez 2005); full points: NGC 6218 stars (Carretta et al. 2006); open squares: High gravity M 3 giants (Sneden et al. 2004); full squares: High gravity M 13 giants (Sneden et al. 2004).

A welcome result from this investigation is that the lithium content of the ejecta of the most massive models, those showing the strongest depletion of oxygen, is $A(\mathrm{Li}) \sim 2$ (see Table 2), and are therefore in excellent agreement with the recent analysis of TO stars in NGC 6752 by Pasquini et al. (2005). Actually, the overall $\mathrm{O}-\mathrm{Li}$ trend in the range $[\mathrm{O} / \mathrm{Fe}]<0.2$ is reproduced (see their Fig. 3).

Although the observed range of magnesium abundances is narrow, so that the existence of a real spread is still debated, our models indicate that a $\mathrm{Mg}-\mathrm{Al}$ anticorrelation should be present, with a spread $\delta[\mathrm{Mg} / \mathrm{Fe}] \sim 0.3 \mathrm{dex}$.

Finally, our models point toward a strong depletion of fluorine, which is strongly burnt at the very beginning of the AGB phase in all models whose initial mass exceeds $4 M_{\odot}$. This agrees with a recent investigation focused on M 4 giants (Smith et al. 2005). A robust confirmation of this scenario would to determine of fluorine abundances in TO and SGB stars.

\section{Conclusions}

We present updated models for the evolution of AGB stars of $3 M_{\odot} \leq M \leq 6.3 M_{\odot}, Y=0.24$, and $Z=0.001$. We test the selfenrichment scenario hypothesis for GCs of intermediate metallicity, comparing the results from spectroscopic investigations of scarcely evolved stars in GCs with the theoretical yields of intermediate mass stars. This task demanded computing of a new set of models, calculated with the latest physical updates concerning the equation of state and the conductive opacities and with an $\alpha$ enhanced mixture to describe self-consistently the chemistry of the first generation of stars that form in the GCs.

The high efficiency of the convective model adopted confirms an important result obtained by this research group regarding the AGB evolution of intermediate mass stars, i.e. the possibility of a strong nucleosynthesis at the bottom of the external convective zone for all the masses $M>3 M_{\odot}$; this HBB also favours a fast increase in the luminosity, a greater mass loss, and therefore reduces the number of thermal pulses experienced by the star during the AGB phase. The present investigation indicates that, for this metallicity a maximum $T_{\text {bce }}=110 \mathrm{MK}$ may be reached for the highest masses. This upper limit, and the strong mass loss suffered by the most massive models, limits the extent of the nucleosynthesis that may be achieved within the envelope.

The combination of HBB and TDU in the most massive models favours a strong depletion of oxygen and fluorine, a modest reduction of magnesium, and a large production of aluminium. Sodium is also produced, via a delicate compromise between production by neon burning and destruction by proton capture. It is confirmed that lithium can be produced at the beginning of the AGB phase via the Cameron-Fowler mechanism, to be later destroyed due to ${ }^{3} \mathrm{He}$ consumption within the envelope. The matter ejected by these models is helium-rich, with a maximum enrichment of $Y=0.35$ found for the $6 M_{\odot}$ model.

On the basis of the results of this work, the strongest point in favour of the self-enrichment scenario is the oxygen depletion, for which both the observations and the theoretical predictions indicate a maximum limit of $[\mathrm{O} / \mathrm{Fe}]=-0.4$; the theoretical oxygen yield is robust, as it turns out to be approximately independent of the details of the mass-loss description, and the relevant cross-sections are known with sufficient accuracy.

The O-Al trend is confirmed by the present investigation, though the extent of the aluminium enrichment of the ejecta is sensitive to the assumptions regarding the cross-sections of the proton capture reactions by the heavy magnesium isotopes and by ${ }^{26} \mathrm{Al}$ : note that the greatest enrichment, $[\mathrm{Al} / \mathrm{Fe}]=1$ is consistent with the aluminium content of the ejecta of the highest masses studied here, which are also those mostly depleted in oxygen. The production of aluminium is associated to magnesium-burning, which we predict to be sligthly depleted, in agreement with the findings of some research groups.

The sodium yield is the most uncertain, due to the huge uncertainties associated to the reactions of the Ne-Na cycle. Our models confirm that, when the maximum allowed values for the reaction rates of the proton capture reaction by ${ }^{22} \mathrm{Ne}$ nuclei are 
adopted, the most oxygen-poor ejecta are also sodium-rich, but the exact extent of the sodium enrichment and confirmation that a clear anticorrelation exists can hardly be fixed with the present cross-sections: the theoretical uncertainties related to the sodium content amount to 0.6 dex.

The yield of lithium and fluorine are most sensitive to the mass-loss rate adopted. With our standard choice, our models predict a O-Li trend that is in excellent agreeent with a recent investigation based on the lithium content of TO stars in NGC 6752. The fluorine content is expected to be extremely poor in any case, the exact abundance being determined by the details of the mass loss description.

\section{References}

Angulo, C., Arnould, M., Rayet, M. et al. 1999, Nucl. Phys. A, 656, 3 Bedin, L. R., Piotto, G., Anderson, J., et al. 2004, ApJ, 605, L125

Bekki, K., Campbell, S. W., Lattanzio, J. C., \& Norris, J. E. 2007, MNRAS, 377, 335

Blöcker, T. 1995, A\&A, 297, 727

Blöcker, T., \& Schönberner, D. 1991, A\&A, 244, L43

Cameron, A. G. W., \& Fowler, W. A. 1971, ApJ, 164, 111

Canuto, V. M. C., \& Mazzitelli, I. 1991, ApJ, 370, 295

Canuto, V. M. C., Goldman, I., \& Mazzitelli, I. 1996, ApJ, 473, 550

Carretta, E. 2006, AJ, 131, 1766

Carretta, E., Gratton, R., Lucatello, S., et al. 2005, A\&A, 433, 597

Carretta, E., Bragaglia, A., Gratton, R. G., et al. 2006, A\&A, 450, 523

Cloutman, L., \& Eoll, J. G. 1976, ApJ, 206, 548

Cohen, J. G., \& Meléndez, J. 2005, AJ, 129, 303

Cottrell, P. L., \& Da Costa, G. S. 1981, ApJ, 245, L79

D'Antona, F., \& Caloi, V. 2004, ApJ, 611, 871

D’Antona, F., \& Caloi, V. 2007, ArXiv e-prints, 709 [arXiv: 0709.4601]

D’Antona, F., \& Mazzitelli, I. 1996, ApJ, 473, 550

D'Antona, F., \& Ventura, P. 2007, MNRAS, 379, 1431

D’Antona, F., Caloi, V., Montalban, J., Ventura, P., \& Gratton, R. 2002, A\&A, 395, 69

D’Antona, F., Cardini, D., Di Mauro, M. P., et al. 2005a, MNRAS, 363, 847

D’Antona, F., Bellazzini, M., Caloi, V., et al. 2005b, ApJ, 631, 868

Decressin, T., Meynet, G., Charbonnel, C., Prantzos, N., \& Ekström, S. 2007, A\&A, 4641029
Denissenkov, P., \& Herwig, F. 2003, ApJ, 590, L99

Denissenkov, P., \& Vandenberg, D. A. 2003, ApJ, 593, 509

Denissenkov, P., \& Weiss, A. 2001, ApJ, 559, L115

Denissenkov, P., Pinsonneault, M., \& Terndrup, D. M. 2006, ApJ, 651, 438

Fenner, Y., Campbell, S., Karakas, A. I., Lattanzio, J. C., \& Gibson, B. K. 2004, MNRAS, 353, 789

Ferguson, J. W., Alexander, D. R., Allard, F., et al. 2005, ApJ, 623, 585 Formicola, A., Imbriani, G., Costantini, H., et al. 2004, Phys. Lett. B, 591, 61 Gratton, R., Bonifacio, P., Bragaglia, A., et al. 2001, A\&A, 369, 87

Gratton, R., Sneden, C., \& Carretta, E. 2004, ARA\&A, 42, 385 Grevesse, N., \& Sauval, A. J. 1998, SSRv, 85, 161

Hale, S. E., Champagne, A. E., Iliadis, C., et al. 2002, Phys. Rev. C, 65, 5801 Hale, S. E., Champagne, A. E., Iliadis, C., et al. 2004, Phys. Rev. C, 70, 5802 Herwig, F. 2005, ARA\&A, 43, 435

Iglesias, C. A., \& Rogers, F. J. 1996, ApJ, 464, 943

Ivans, I. I., Sneden, C., Kraft, R. P., et al. 1999, AJ, 118, 1273

Izzard, R. G., Lugaro, M., Karakas, A. I., Iliadis, C., \& van Raai, M. 2007, A\&A, 466, 641

Kraft, R. P. 1994, PASP, 106, 553

Lee, Y.-W., Joo, S.-J., Han, S.-I., et al. 2005, ApJ, 621, L57

Maeder, A., \& Meynet, G. 2006, A\&A, 448, L37

Mazzitelli, I., D’Antona, F., \& Ventura, P. 1999, A\&A, 348, 846

Pasquini, L., Bonifacio, P., Molaro, P., et al. 2005, A\&A, 441, 549

Piotto, G., Villanova, S., Bedin, L. R., et al. 2005, ApJ, 621, 777

Piotto, G., Bedin, L. R., Anderson, J., et al. 2007, ApJ, 661, L53

Prantzos, A., \& Charbonnel, C. 2006, A\&A, 458, 135

Ramirez, S. V., \& Cohen, J. G. 2003, AJ, 125, 224

Sackmann, I. J., \& Boothroyd, A. I. 1992, ApJ, 392, L71

Saumon, D., Chabrier, G., \& Van Horn, H. M. 1995, ApJS, 99, 713

Shönberner, D. 1979, A\&A, 79, 108

Smith, V. V., Cunha, K., Ivans, I. I., et al. 2005, ApJ, 633, 392

Sneden, C., Kraft, R. P., Guhathakurta, P., Peterson, R. C., \& Fulbright, J. P. 2004, AJ, 127, 2162

Stolzmann, W., \& Blöecker, T. 2000, A\&A, 361, 1152

Vassiliadis, E., \& Wood, P. R. 1993, ApJ, 413, 641

Ventura, P., \& D’Antona, F. 2005a, A\&A, 431, 279

Ventura, P., \& D'Antona, F. 2005b, A\&A, 439, 1075

Ventura, P., \& D'Antona, F. 2006, A\&A, 457, 995

Ventura, P., Zeppieri, A., D’Antona, F., \& Mazzitelli, I., 1998, A\&A, 334, 953

Ventura, P., D'Antona, F., \& Mazzitelli, I. 2000, A\&A, 363, 605

Ventura, P., D’Antona, F., Mazzitelli, I., \& Gratton, R. 2001, ApJ, 550, L65

Ventura, P., D’Antona, F., \& Mazzitelli, I. 2002, A\&A, 393, 215

Vitense, E. 1953, Zs. Ap., 32, 135 